\section{RMD Open}

Rheumatic \&

Musculoskeletal Diseases

\title{
Systemic lupus erythematosus; stroke and myocardial infarction risk: a systematic review and meta-analysis
}

Jinoos Yazdany, ${ }^{1}$ Nick Pooley, ${ }^{2}$ Julia Langham, ${ }^{3}$ Lindsay Nicholson, ${ }^{2}$ Sue Langham, ${ }^{4}$ Nina Embleton, ${ }^{5}$ Xia Wang, ${ }^{6}$ Barnabas Desta, ${ }^{7}$ Volkan Barut, ${ }^{8}$ Edward Hammond ${ }^{9}$

To cite: Yazdany J, Pooley N, Langham J, et al. Systemic lupus erythematosus; stroke and myocardial infarction risk: a systematic review and meta-analysis. RMD Open 2020;6:e001247. doi:10.1136/ rmdopen-2020-001247

- Supplemental material is published online only. To view please visit the journal online (http://dx.doi.org/10.1136/rmdo pen-2020-001247)

Received 20 March 2020 Revised 25 June 2020 Accepted 11 July 2020

\section{Check for updates}

(c) Author(s) (or their employer(s)) 2020. Re-use permitted under CC BY-NC. No commercial re-use. See rights and permissions. Published by BMJ.

For numbered affiliations see end of article.

Correspondence to Barnabas Desta; barnabas.desta@astrazeneca. com

\section{ABSTRACT}

Objective To evaluate the risk of stroke and myocardial infarction (MI) in adult patients with systemic lupus erythematosus (SLE) through a systematic review and meta-analysis.

Methods We searched MEDLINE and EMBASE from inception to May 2020 to identify observational studies (cohort and cross-sectional) that evaluated risk of stroke and $\mathrm{MI}$ in adult patients with SLE compared with the general population or healthy controls. Studies were included if they reported effect-size estimates that could be used for calculating pooled-effect estimates. Random-effects models were used to calculate pooled risk ratios (RRs) and $95 \%$ Cls for stroke and Ml. Heterogeneity quantified by the $\mathrm{I}^{2}$ test and sensitivity analyses assessed bias.

Results In total, 26 studies were included in this metaanalysis: 14, 5 and 7 studies on stroke, Ml and both stroke and $\mathrm{MI}$, respectively. The pooled RR for ischaemic stroke was 2.18 (95\% Cl 1.78 to $\left.2.67 ; I^{2} 75 \%\right)$, intracerebral haemorrhage 1.84 (95\% Cl 1.16 to $\left.2.90 ; I^{2} 67 \%\right)$, subarachnoid haemorrhage $1.95\left(95 \% \mathrm{Cl} 0.69\right.$ to $5.52 ; \mathrm{I}^{2}$ $94 \%)$, composite stroke 2.13 (95\% Cl 1.73 to $\left.2.61 ; I^{2} 88 \%\right)$ and $\mathrm{Ml} 2.99$ (95\% Cl 2.34 to $\left.3.82 ; I^{2} 85 \%\right)$. There was no evidence for publication bias, and sensitivity analyses confirmed the robustness of the results.

Conclusions Overall, patients with SLE were identified to have a twofold to threefold higher risk of stroke and MI. Future research on the interaction between known SLEspecific modifiable risk factors and risk of stroke and MI to support development of prevention and treatment strategies are needed.

PROSPERO registration number CRD42018098690.

\section{INTRODUCTION}

Systemic lupus erythematosus (SLE) is a chronic autoimmune disorder characterised by alternating periods of flares and remission, and irreversible organ damage associated with disease activity. ${ }^{1}$ The skin, joints, heart, kidneys, central nervous system and haematologic system are some of the most commonly affected organs. $^{2}{ }^{3}$ Organ damage has been associated with increased morbidity and mortality. ${ }^{4}$ Although recent data suggest that

\section{Key messages}

What is already known about this subject?

- Systemic lupus erythematosus (SLE) is a chronic autoimmune disorder associated with increased comorbidities.

- Epidemiological studies have suggested an increased risk of cardiovascular events, including stroke and myocardial infarction (MI), in patients with SLE.

What does this study add?

- The pooled relative risk of stroke (intracerebral haemorrhage, ischaemic stroke and subarachnoid haemorrhage) and MI from epidemiological studies was found to be twofold to threefold higher among patients with SLE compared with the general population or healthy controls.

- This magnitude of risk was 1.84-fold higher for intracerebral haemorrhage, 1.95-fold for subarachnoid haemorrhage, 2.13-fold for composite stroke, 2.18-fold for ischaemic stroke and 2.99-fold for MI.

How might this impact clinical practice?

- This study confirms the need for treatment strategies that consider prevention and treatment of modifiable cardiovascular risk factors in addition to SLE management.

mortality decreased in patients with SLE over the last 30 years, mortality due to cardiovascular disease (CVD) has remained high, ${ }^{5-8}$ an estimated twofold to threefold increased risk of CVD-associated mortality compared with the general population. ${ }^{9-11}$

Stroke and myocardial infarction (MI) are major CVD events that are potentially lifethreatening. ${ }^{12}$ Understanding the magnitude of stroke and MI risk in patients with SLE and characterising patients at highest risk would support the development of strategies for preventing and treating or modifying risk factors. Patients with SLE have an increased risk of stroke $^{10}{ }^{13}$ and MI. ${ }^{10}$ Evidence includes a meta-analysis of cohort studies published 
prior to 2015 that compared patients with SLE with the general population. ${ }^{13}$ There are no recent meta-analyses that evaluate both stroke and MI across multiple observational study types to estimate pooled risk.

We aimed to synthesise evidence from published observational studies reporting risk of major cardiovascular events in adults with SLE compared with the general population or healthy controls. ${ }^{14}$ We report our findings on the risk of stroke and MI in patients with SLE. We also evaluate the role of age and sex in stroke and MI risk.

\section{METHODS}

\section{Search strategy}

This study was conducted in accordance with the Metaanalysis of Observational Studies in Epidemiology and the Preferred Reporting Items for Systematic reviews and Meta-Analyses guidelines for conducting and reporting systematic reviews. ${ }^{15} 16$ The study protocol was prepared and published via the International Prospective Register of Systematic Reviews, PROSPERO (\#CRD42018098690). ${ }^{14}$ Searches for full-text reports containing original data were run in Ovid MEDLINE and EMBASE until March 2018; an additional update search was run until May 2020. The detailed search strategy is available in online supplemental table S1. We also searched the reference lists of articles and contacted experts in the field.

\section{Eligibility criteria}

We included full publications of observational studies (cohort and cross-sectional studies) published in English reporting the risk of CVD outcomes in adult patients with SLE compared with the general population or healthy controls. Patients with SLE were identified by International Classification of Diseases (ICD) codes, American College of Rheumatology (ACR) criteria or clinician-confirmed diagnosis. ${ }^{17} 18$ The outcomes reported in this manuscript include fatal and non-fatal stroke (including subtypes) and MI events. Studies were included if they reported one of the following measures of relative risk: HR, rate ratio, risk ratio (RR), OR, incidence rate ratio, proportionate morbidity ratio, standardised mortality rate or standardised incidence rate with 95\% CIs. Abstracts of unpublished studies were excluded as data were not reported to support formal comparison.

\section{Screening and abstraction process}

Two-stage screening (title/abstract and full-text screening), data extraction and risk of bias assessment were performed independently by two reviewers (NP and $\mathrm{LN})$; disagreement was resolved by consensus involving a third reviewer (JL). Studies that met the eligibility criteria and reported original data were included in the review. Data on study characteristics and the effect measure for outcomes of interest (fatal and non-fatal events) were extracted.

\section{Risk of bias and quality assessment}

The risk of bias of included studies was estimated using the Newcastle-Ottawa Scale ${ }^{19}$ and an SLE-specific 12point scale developed for use in previous SLE systematic reviews. ${ }^{8} 111320-23$ The SLE-specific 12-point scale scores quality in five domains: (1) source of study sample (population-based and clinic-based), (2) cohort type (inception and non-inception), (3) SLE definition (ACR classification criteria for SLE, ICD codes and medical record review), (4) length of SLE exposure ( $\geq 10$ or $<10$ years, $\geq 5$ or $<5$ years or not defined) and (5) ascertainment of outcome (medical record review, ICD code only and exclusion of prevalent outcomes at baseline) (online supplemental table S2). The Newcastle-Ottawa Scale assesses study quality in three domains: (1) selection of study groups, (2) comparability of cohorts by design or analysis and (3) ascertainment of outcomes of interest (online supplemental table S3). Studies were classified as having low, moderate or high risk of bias based on results from domains in both scales.

\section{Statistical analysis}

We performed meta-analyses for stroke and MI where two or more studies with a low risk of bias reported usable data. One study was selected for inclusion in the metaanalysis based on study quality, population size and length of study period if there were two studies that reported findings from overlapping populations.

ORs, HRs, rate ratios, standardised incidence ratios and standardised mortality ratios were considered equal estimates assuming rare occurrence ${ }^{24}$ and referred to as 'risk ratios' throughout this publication. The most adjusted RR was used. A DerSimonian and Laird ${ }^{25}$ randomeffects model was fit to calculate the pooled RR and 95\% CIs for all outcomes.

Heterogeneity was measured using the Cochran's $Q$ statistic with statistical significance set at $p<0.10$ and quantified by the $\mathrm{I}^{2}$ test. Publication bias was assessed with both funnel plots and the Egger's test. ${ }^{26}$

Robustness of the results was assessed by the leavelout function, ${ }^{27}$ which examined the effect of removing individual studies on pooled estimates. Several sensitivity analyses were performed, including least-adjusted analysis, studies published during or after 2014, studies published before 2014, studies with low risk of bias, studies reporting non-fatal events, studies reporting non-fatal/fatal events, studies previously excluded because the populations overlapped with another study; and excluding cross-sectional studies. All analyses were conducted in $\mathrm{R}$ version 3.5.1 using the packages metafor and forestplot.

We describe reported RRs for the patient subgroups of age and sex, for which data were available from specific studies. Due to the paucity of data, no meta-analyses were conducted for subgroups.

\section{Patient and public involvement}

No patients or the public were involved in setting the research question or outcome measures, nor in the 
design and implementation of the study. However, the dissemination plan targets a wide audience including members of the public, patients, health professionals and experts in the speciality through various channels including peer-reviewed publications and conference posters and presentations.

\section{RESULTS}

\section{Literature search}

The original search of the two electronic databases identified 3252 records; 2569 articles remained after duplicates were removed. Of these, 2400 were excluded after screening titles and abstracts. After full-text review, 23 publications reporting on stroke and MI were retained for inclusion in this report (figure 1). The updated search identified 612 records; 420 articles remained after duplicates were removed. Of these, 372 were excluded after screening titles and abstracts. After full-text review, three additional publications reporting on stroke and MI were retained, bringing the total for inclusion to 26 publications. A list of excluded studies, with reasons, is outlined in online supplemental table S4.

\section{Study characteristics}

Characteristics of the 26 included studies ${ }^{5-7} 28-50$ are summarised in table 1 . There were 23 cohort studies and three cross-sectional studies.

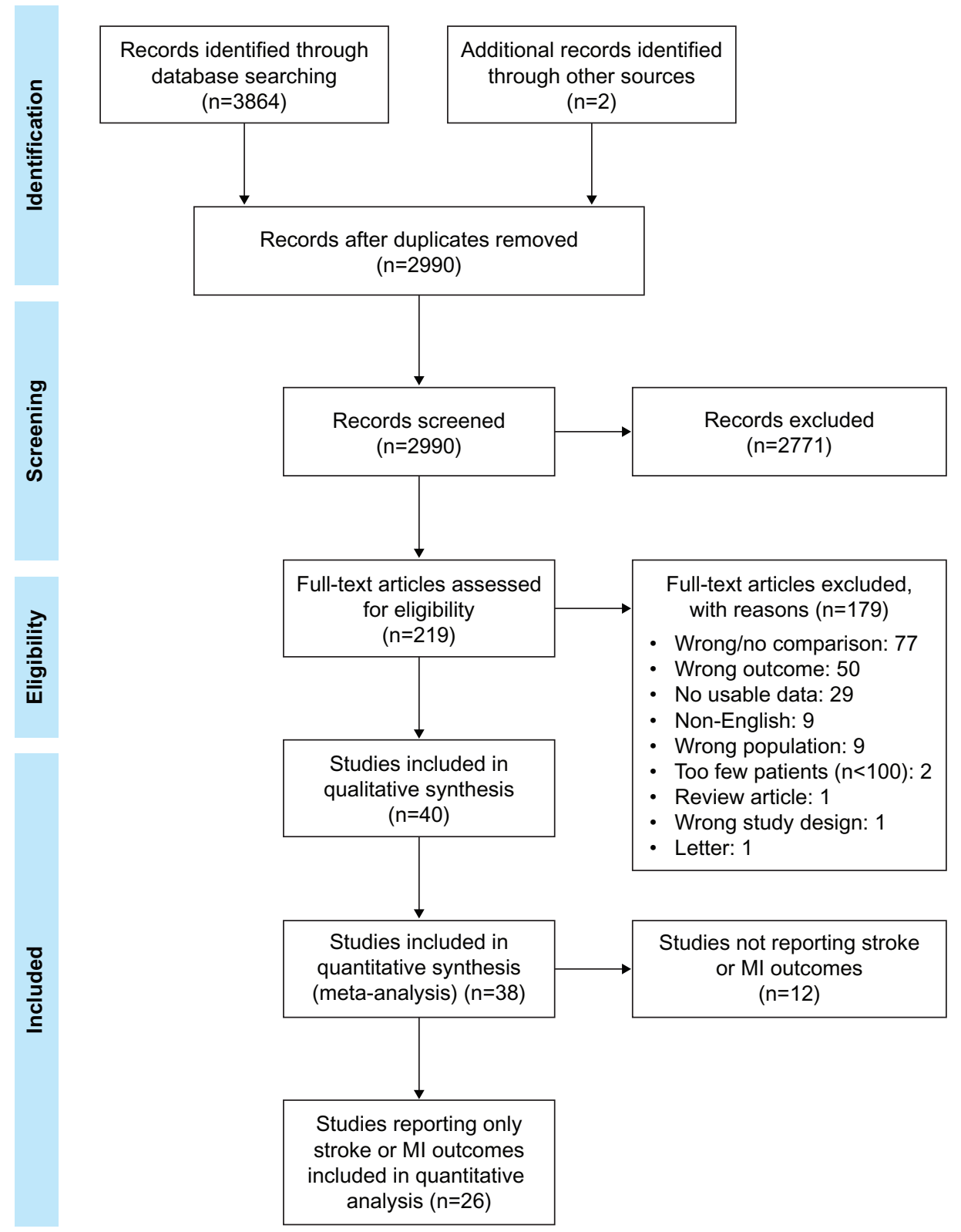

Figure 1 Flow diagram of the systematic literature review process to evaluate the risk of stroke and MI in patients with SLE compared with the general population or healthy controls. MI, myocardial infarction; SLE, systemic lupus erythematosus. 


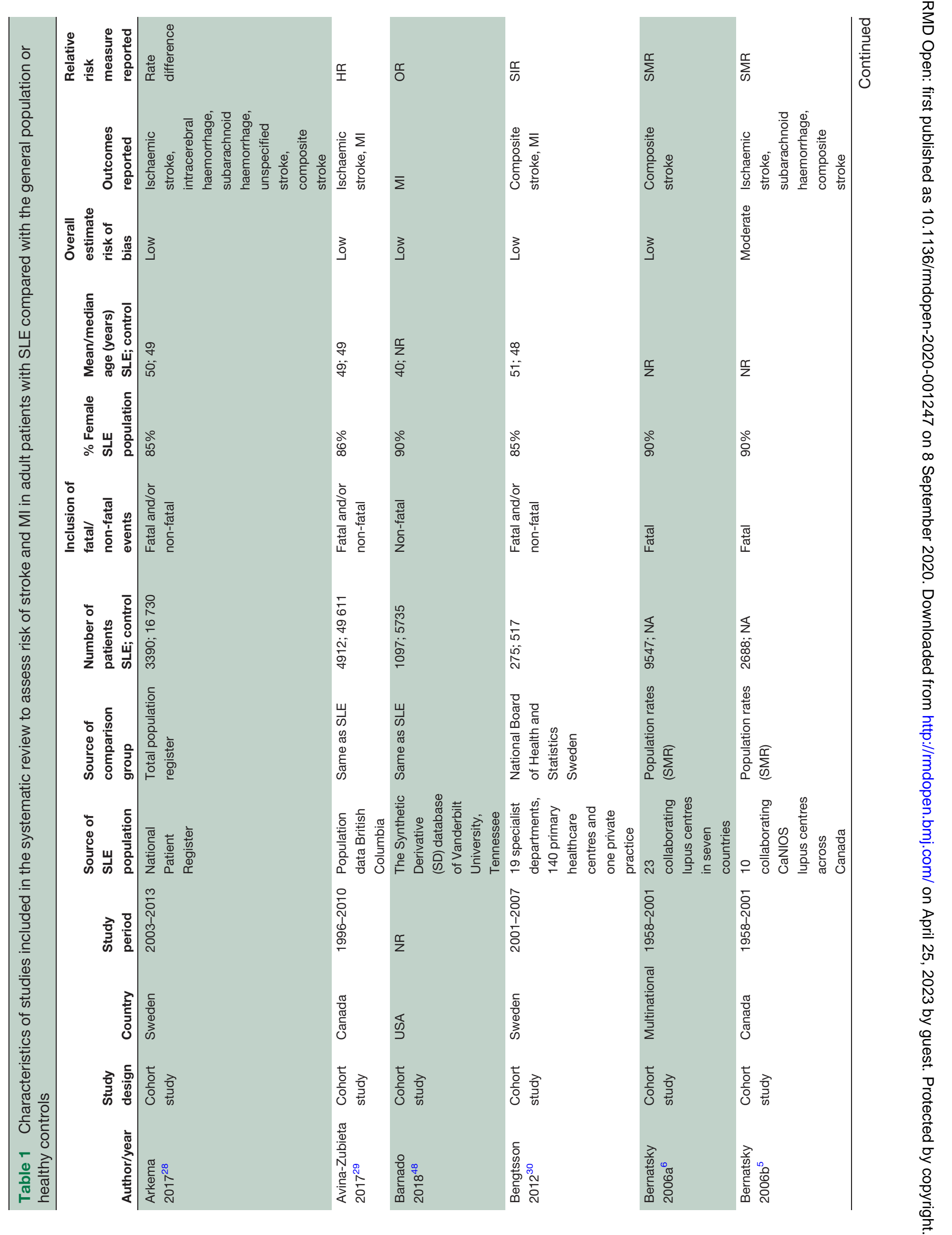




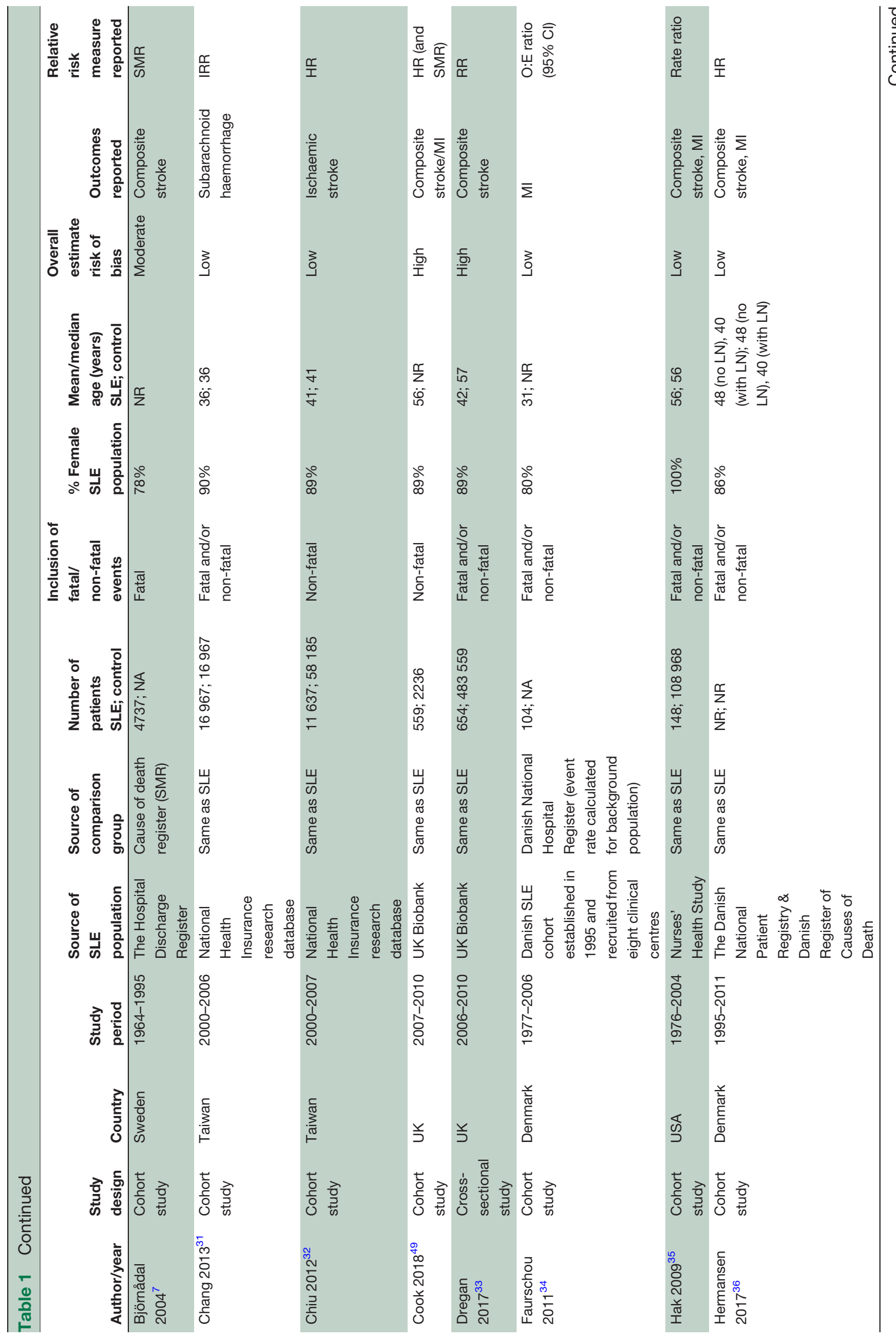




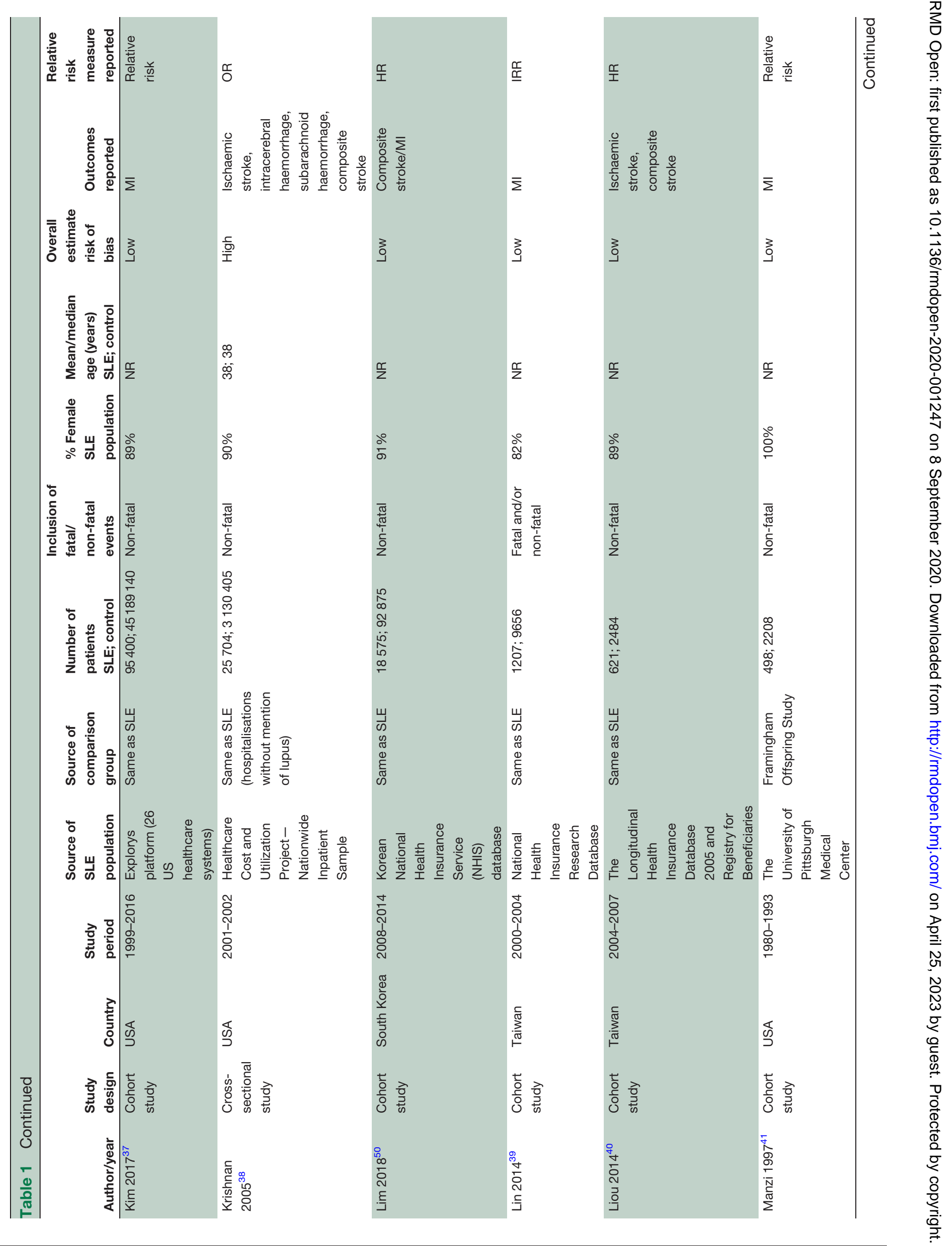




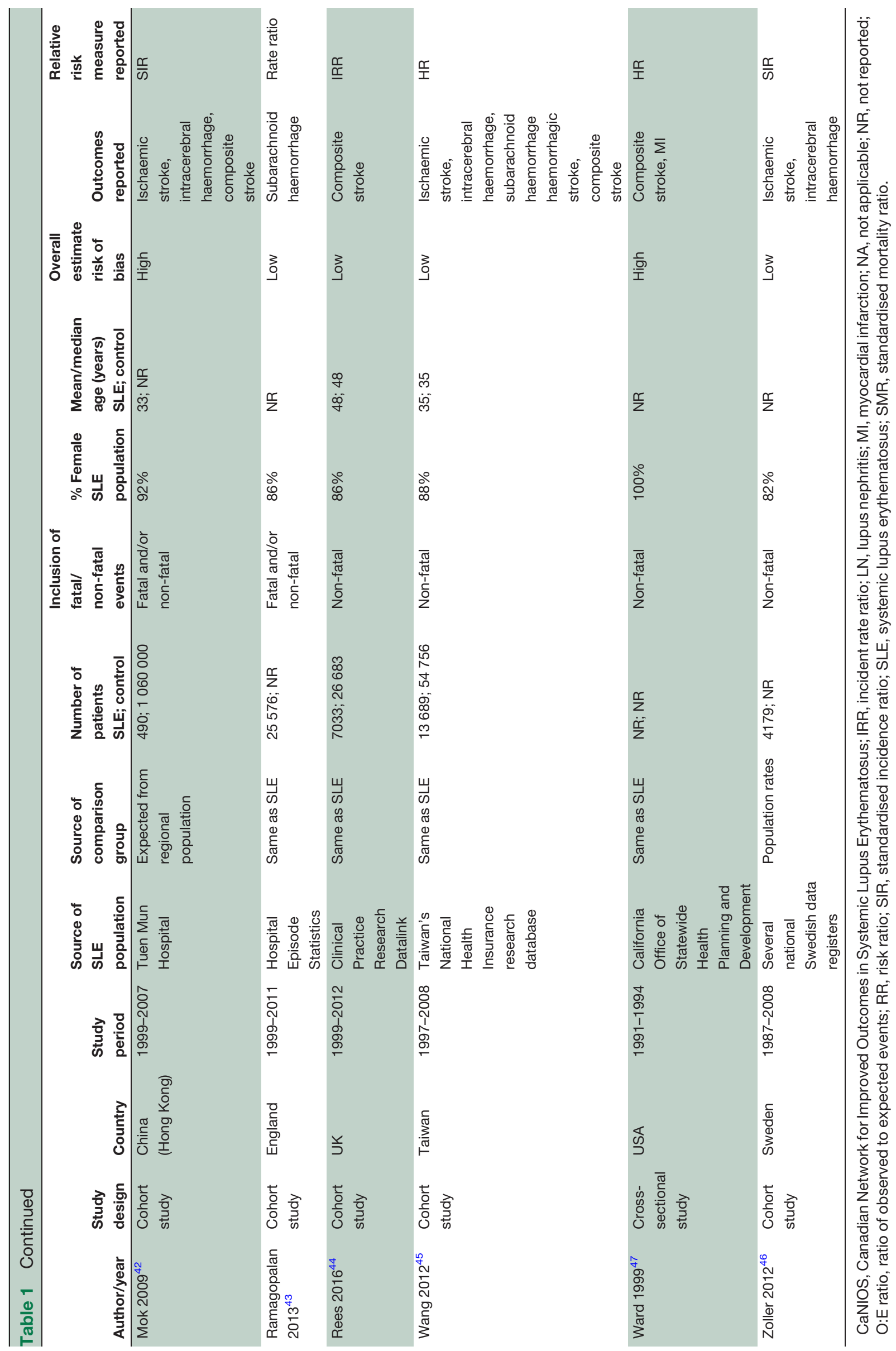


Twenty-four studies reported the number of patients with SLE assessed ( $\mathrm{N}=249687)$ and 15 studies reported the number of general population/healthy controls assessed ( $\mathrm{N}=50310715)$. Studies were conducted in Asia $(n=7)$, Europe $(n=10)$, North America $(n=8)$ or multiple countries (including centres in Europe, North America and Asia; $n=1)$. Study durations ranged from 1 to 43 years. The percentage of female patients ranged from $78 \%$ to $100 \%$. Average age, reported in 15 studies, ranged from 31 to 56 years. Bias was assessed to be low in 19 studies $^{6}$ 28-32 34-37 39-41 43-46 and as moderate in two studies. ${ }^{57}$ Five studies were assessed as having high risk of bias, three of which were cross-sectional studies ${ }^{33847}$ and two were cohort studies. ${ }^{42} 49$ The risk of bias assessment for included studies is summarised in online supplemental table S5.

\section{Stroke}

Meta-analyses were performed for the following stroke outcomes: composite stroke, subarachnoid haemorrhage, intracerebral haemorrhage and ischaemic stroke. No meta-analysis was performed for haemorrhagic stroke $(n=1)$ and unspecified stroke $(n=2)$, only one of the two studies had low risk of bias.

\section{Composite stroke}

Composite stroke was reported in 16 studies: six studies evaluated fatal or non-fatal events, ${ }^{28} 30 \quad 33 \quad 353642$ five evaluated non-fatal events ${ }^{38} 40444547$ and five evaluated fatal events. ${ }^{5-7}$ Five studies were not included in the main meta-analysis: four had overlapping populations with other studies 5303340 and one only reported data by age group. ${ }^{47}$ Nine of the 11 studies included had low risk of bias (online supplemental table S5).

Composite stroke was identified by ICD-8, ICD-9 and ICD-10 codes in 12 of 16 studies. In the remaining three studies, stroke was identified by unreported read codes, physician diagnosis, National Survey of Stroke criteria or Biobank database based on ICD-10 codes (online supplemental table S6). The ICD codes used to create the composite stroke endpoint were specific to each study and are listed in online supplemental table S6.

SLE was associated with an increased risk of composite stroke, with a pooled RR of 2.13 (95\% CI 1.73 to 2.61; I $^{2}$ for heterogeneity $88.3 \%$; $\mathrm{df}=10 ; \mathrm{p} \leq 0.001$ ) (figure $2 \mathrm{~A}$ ).

\section{Subarachnoid haemorrhage}

Subarachnoid haemorrhage was reported in six studies: three studies evaluated fatal/non-fatal events, ${ }^{28} 3143$ two studies evaluated non-fatal events ${ }^{38} 45$ and one study evaluated fatal events. ${ }^{5}$ Two studies were not included in the meta-analysis because they did not provide usable $95 \%$ CIs. ${ }^{531}$ Three of the four studies included had low risk of bias (online supplemental table S5).

Subarachnoid haemorrhage was identified by ICD-8, ICD-9 and ICD-10 codes in all studies. The ICD codes used were the same in all studies that reported them (online supplemental table S6).
Risk of subarachnoid haemorrhage did not significantly increase in patients with SLE, with a pooled RR of 1.95 (95\% CI 0.69 to 5.52 ; $\mathrm{I}^{2}$ for heterogeneity $94.4 \%$; $\mathrm{df}=3$; $\mathrm{p}<0.001$ ) (figure $2 \mathrm{~B}$ ).

\section{Intracerebral haemorrhage}

Intracerebral haemorrhage was reported in five studies: two studies evaluated fatal/non-fatal events ${ }^{28}{ }^{42}$ and three studies evaluated non-fatal events. ${ }^{38} 4546$ One study was not included in the meta-analysis because the population overlapped with another study. ${ }^{46}$ Of the four studies included, two had low risk of bias (online supplemental table S5).

Intracerebral haemorrhage was identified by ICD-8, ICD-9 and ICD-10 codes in four of five studies. The codes used were similar in all studies that reported them and are listed in online supplemental table S6. In one study, physician diagnosis confirmed case identification.

SLE was associated with an increased risk of intracerebral haemorrhage, with a pooled RR of 1.84 (95\% CI 1.16 to $2.90 ; \mathrm{I}^{2}$ for heterogeneity $67.4 \%$; $\mathrm{df}=3 ; \mathrm{p}<0.0027$ ) (figure 2C).

\section{Ischaemic stroke}

Ischaemic stroke was reported in nine studies: three studies evaluated fatal/non-fatal events, ${ }^{28} 2942$ five studies evaluated non-fatal events ${ }^{32} 38404546$ and one study evaluated fatal events. ${ }^{5}$ Four studies were not included in the meta-analysis, two of which had overlapping study populations, ${ }^{45}{ }^{46}$ one did not report a usable $95 \% \mathrm{CI}^{5}$ and one reported data only for a subpopulation. ${ }^{40}$ Of the five studies included, three had low risk of bias (online supplemental table S5).

Ischaemic stroke was identified by ICD-8, ICD-9 and ICD-10 codes in seven of nine studies. The codes used were similar in all studies that reported them and are listed in online supplemental table S6. In the remaining studies, physician diagnosis or national insurance claims data confirmed case identification.

SLE was associated with an increased risk of ischaemic stroke, with a pooled RR of 2.18 (95\% CI 1.78 to $2.67 ; \mathrm{I}^{2}$ for heterogeneity $75.4 \%$; $\mathrm{df}=4 ; \mathrm{p} \leq 0.001$ ) (figure 2D).

\section{Myocardial infarction}

MI was reported in 12 studies: six studies evaluated fatal/ non-fatal events ${ }^{29} 30$ 34-36 39 and six studies evaluated nonfatal events. ${ }^{37} 4147-50$ Four studies were not included in the meta-analysis: two because they reported data only for a subpopulation, ${ }^{4147}$ one because it only reported data on lupus nephritis $(\mathrm{LN})^{34}$ and one owing to population overlap with another study. ${ }^{30}$ Of the eight studies included, all but one had low risk of bias ${ }^{49}$ (online supplemental table S5).

MI was identified by ICD-8, ICD-9 and ICD-10 codes in 8 of 12 studies. The codes used were similar in all studies that reported them and are listed in online supplemental table S6. In the remaining studies, a combination of WHO criteria, hospital data, Biobank 

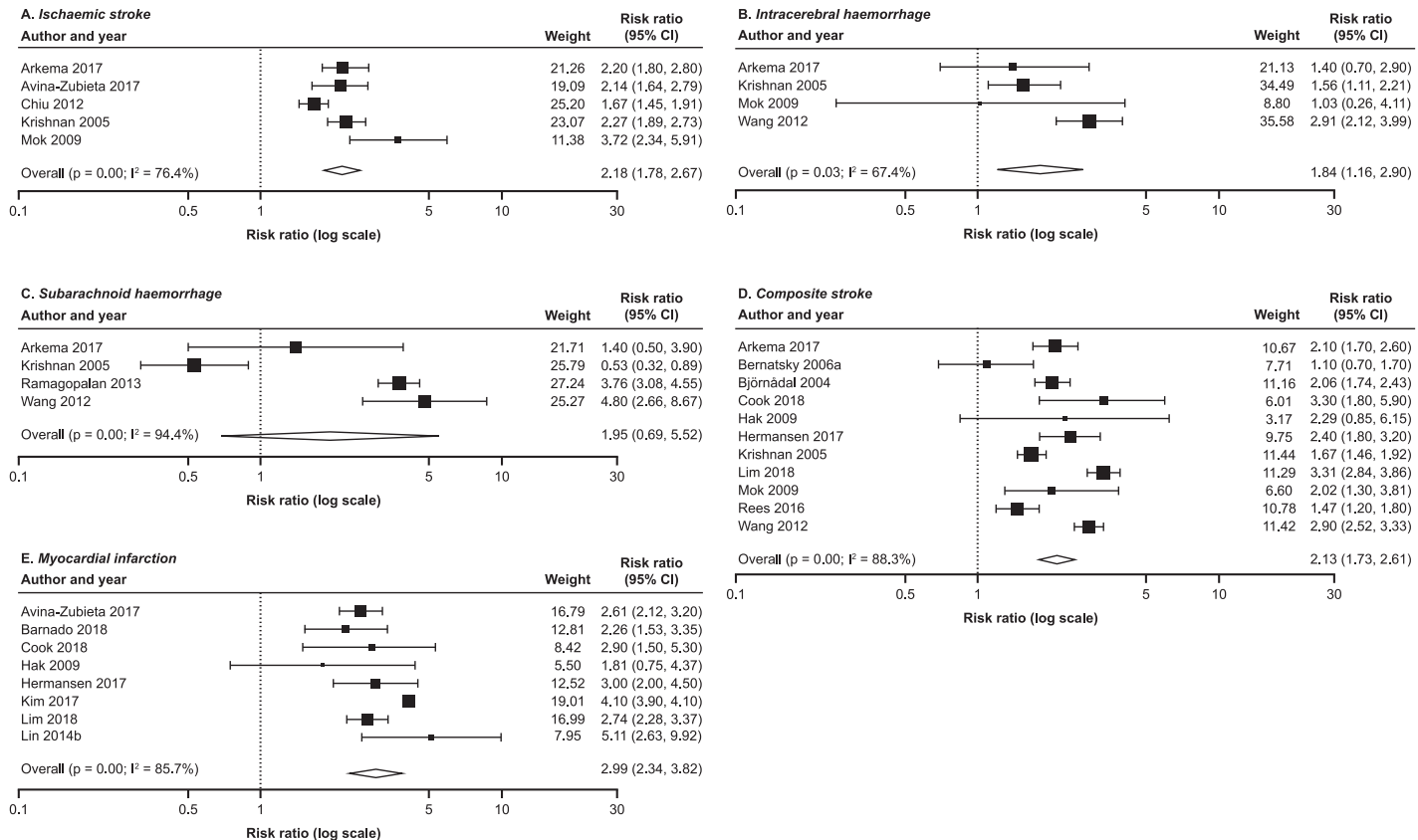

Risk ratio (log scale)

Figure 2 Forest plots of pooled risk ratios for stroke and Ml outcomes in adult patients with SLE compared with the general population or healthy controls: (A) composite stroke, (B) subarachnoid haemorrhage, (C) intracerebral haemorrhage, (D) ischaemic stroke, (E) MI. MI, myocardial infarction; SLE, systemic lupus erythematosus.

database based on ICD-10 codes or autopsy evidence confirmed identification. One study did not report how MI was identified.

SLE was associated with an increased risk of MI, with a pooled RR of 2.99 (95\% CI 2.34 to 3.82 ; I $^{2}$ for heterogeneity $85.7 \%$; $\mathrm{df}=7 ; \mathrm{p} \leq 0.001$ ) (figure $2 \mathrm{E}$ ).

\section{Sensitivity analyses and heterogeneity}

The leavelout method and various sensitivity analyses confirmed the robustness of the results (table 2 , online supplemental table $\mathrm{S} 7$ ).

In terms of the leavelout function, only one analysis showed a statistically significant effect of removing an individual study. Removal of a cross-sectional study ${ }^{38}$ evaluating subarachnoid haemorrhage increased the relative risk identified in the base case from 1.95 to 3.06 (RR 3.06, $95 \%$ CI 1.87 to 5.02 ).

The base-case analyses identified composite stroke, subarachnoid haemorrhage, intracerebral haemorrhage, ischaemic stroke and MI as being statistically significantly increased in persons with SLE compared with the general population. Results of all sensitivity analyses for composite stroke remained significant. For subarachnoid haemorrhage, intracerebral haemorrhage and ischaemic stroke, a number of sensitivity analyses resulted in relative risks that were, in general, higher than the base case but statistically non-significant. We observed this when sensitivity analyses were restricted to studies reporting only on non-fatal/fatal events, only low risk of bias studies, only studies published during or after 2014 and excluding cross-sectional studies. For MI, three of the sensitivity analyses resulted in a lower relative risk that was not statistically significant (including only studies reporting on non-fatal/fatal events, only studies published before 2014 and only studies reporting on non-fatal events).

Visual examination of the funnel plots showed evidence of publication bias, which was supported by the Egger's test for ischaemic stroke $(p=0.001)$ but not for composite stroke $(p=0.885)$, subarachnoid haemorrhage $(p=0.686)$, intracerebral haemorrhage $(p=0.265)$ and MI $(\mathrm{p}=0.500)$.

\section{Subgroup evaluation of age and sex}

Eight studies reported the relative risk of stroke stratified by age. ${ }^{28} 29364244-47$ Data suggest that risk of stroke increases with age in SLE and non-SLE populations. Patients with SLE have a higher relative risk of stroke, particularly in younger age groups, compared with agematched population controls. In patients with SLE aged $<30$ years, RRs ranged from 14.5 to 53.9 , and in patients aged $>70$ years, RRs ranged from 0.53 to 1.76 , depending on type of stroke and study design (online supplemental figure $\mathrm{S} 1$ ).

Four studies reported the relative risk of MI stratified by age; however, differences in age group boundaries and small numbers of patients with SLE within groups meant that the data could not be summarised. ${ }^{29} 304147$ It was not possible to summarise the relative risk of stroke and MI stratified by sex due to the small numbers of male patients included in the studies. 


\section{DISCUSSION}

In this meta-analysis of 26 real-world observational studies, patients with SLE had a twofold increase in risk of stroke and threefold increase in risk of MI compared with the general population or healthy controls. To our knowledge, this is the first meta-analysis to assess the risk of both stroke and MI across multiple observational study types in adult patients with SLE compared with the general population or healthy controls.

Rheumatologists are increasingly recognising the risk of CVD as a comorbid disease in patients with SLE. Recent 2019 guidelines from the EULAR recommend assessment of CVD risk and initiation of preventive strategies for patients with SLE when necessary. ${ }^{51}$ Because the development of CVD could result in decreased health-related quality of life $\mathrm{e}^{52}$ and early mortality, ${ }^{95}$ health education and risk factor modification are important for this patient population.

Our findings are consistent with a published metaanalysis for stroke in patients with SLE that included studies up to June $2015 .{ }^{13}$ The increased risk of composite stroke (RR 2.13), intracerebral haemorrhage (RR 1.84) and ischaemic stroke (RR 2.18) are consistent with findings reported by Holmqvist et al. ${ }^{13}$ However, the higher number of studies included in our meta-analysis meant increased precision, evidenced by smaller CIs. The increased risk of subarachnoid haemorrhage (RR 1.95) in our analysis is lower than that reported by Holmqvist et $^{13}{ }^{13}$ (RR 3.85) because our analysis includes a cross-sectional study published in 2005 that reports a low RR (0.53). ${ }^{38}$ When we excluded this study in the sensitivity analysis, the RR increased to 3.50 (95\% CI 2.24 to 5.48$)$, similar to that reported by Holmqvist et al. ${ }^{13}$

Our analyses confirm that the relative risk of stroke is higher in younger patients with SLE compared with agematched controls and corroborate findings from a previous systematic review. ${ }^{13}$ Although the underlying pathogenesis of increased stroke risk in patients with SLE is the subject of ongoing research, accelerated atherosclerosis likely plays a role. ${ }^{54-56}$ Accelerated atherosclerosis has also been shown to be associated with LN, which often develops at a young age and is also associated with increased CVD risk. ${ }^{57}{ }^{58} \mathrm{~A}$ previous study found that patients with SLE and a history of LN had twice the rate of carotid plaque as age-matched patients with SLE without LN. Patients with SLE and no LN did not differ from age-matched non-SLE controls regarding carotid plaques. ${ }^{58}$ Accelerated atherosclerosis is often considered to be the primary cause of increased CVD risk in patients with SLE. ${ }^{59}$

Our study is strengthened by a rigorous methodological approach based on international guidelines for conduct and reporting of systematic reviews and meta-analyses. The study design included a comprehensive search of multiple databases, reducing the likelihood of omitting evidence reported in key studies. The study selection criteria ensured that studies with overlapping populations were evaluated only once, ensuring greater confidence in reported relative risk estimates. Our study has some limitations. We identified heterogeneity across the evaluated studies that may be a result of variations in population characteristics, control group selection and risk measure reported. An additional source of heterogeneity may result from the extent to which the SLE and comparison populations were matched for CVD risk factors. Some studies matched the population for a wide range of risk factors or adjusted for them in the analysis, while others only matched or adjusted for a limited number of risk factors. However, multiple sensitivity analyses confirmed the increased risk of stroke and MI in patients with SLE. Because of limited data, meta-analyses could not be performed on patient subgroups.

In addition to age, other known MI and stroke risk factors and SLE-related factors are likely to be important in explaining the observed elevated risk. Of SLE-related factors, disease duration and damage, antiphospholipid antibodies, renal and neuropsychiatric disease and steroids have been linked to increased risk of CVD events. ${ }^{59} 60$ In this work, although subgroup analyses including these risk factors were not possible owing to limited data, our meta-analysis included two studies that suggest an association between CVD risk and treatment type. ${ }^{33}{ }^{40}$ In one study, the risk of ischaemic stroke was stratified by steroid use, and a statistically significant increase in relative risk was identified only in patients with concomitant steroid use. ${ }^{40} \mathrm{~A}$ more recent study stratified the relative risk of stroke and venous thromboembolism into four treatment subgroups: no therapy, disease-modifying antirheumatic drugs (DMARDs), non-steroidal antiinflammatory drugs (NSAIDs) and corticosteroids. ${ }^{33}$ The relative risk of composite stroke was shown to be highest in those treated with NSAIDs or corticosteroids followed by those treated with DMARDs. However, these differences were not statistically significant. In addition, our meta-analysis included one study that investigated the effects of end-stage renal disease (ESRD) on the relative risk of ischaemic heart disease (IHD) in patients with SLE and identified a higher relative risk of IHD in patients with ESRD. ${ }^{34}$

A future synthesis of the available evidence for specified subgroups among patients with SLE would be useful in highlighting potential modifiable risk factors for CVD.

\section{CONCLUSION}

The risk of stroke and MI events among adult patients with SLE is twofold to threefold higher compared with the general population or healthy controls. Known MI and stroke risk factors and SLE-related factors are likely to be associated with the observed elevated risk. Understanding the various mechanisms underlying increased CVD risk in patients with SLE, including how antiphospholipid antibodies or antiphospholipid syndrome may modify this risk, will support prevention and treatment strategies and advance informed patient and physician decisions. 


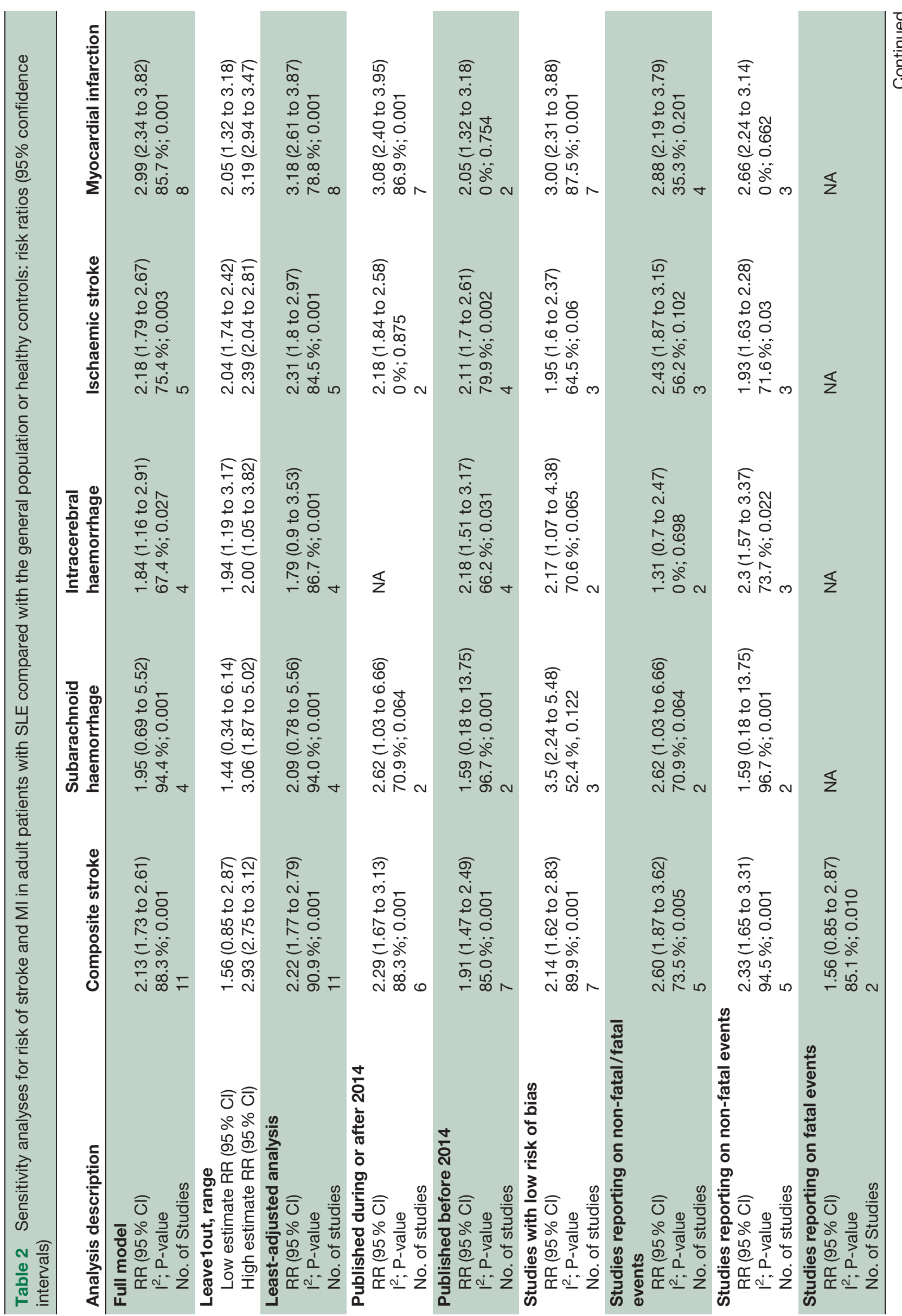




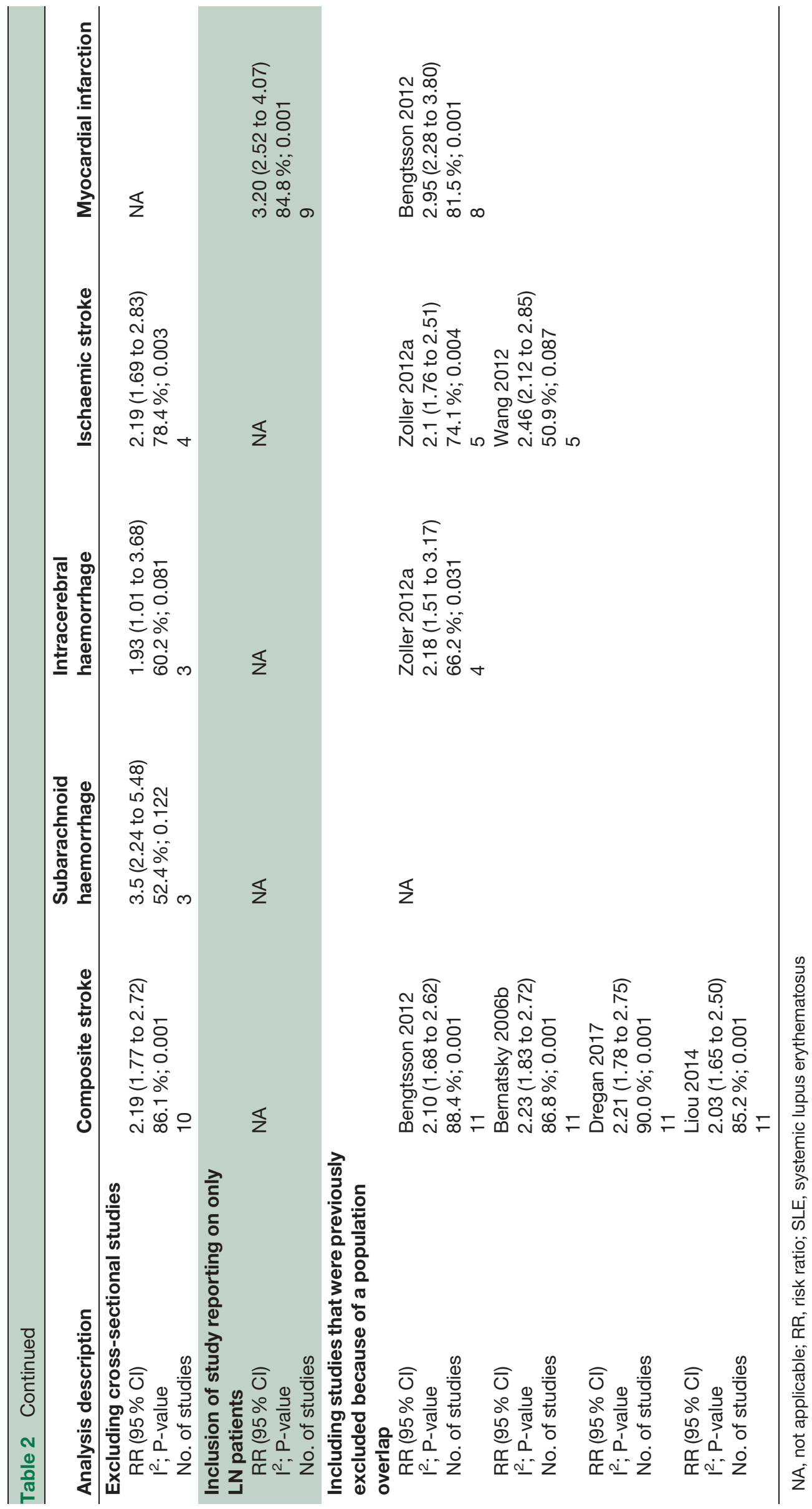


Author affiliations

${ }^{1}$ Division of Rheumatology, Department of Medicine, University of California, San Francisco, California, USA

${ }^{2}$ Systematic Review Group, Maverex Limited, Manchester, UK

${ }^{3}$ Epidemiology Group, Maverex Limited, Manchester, UK

${ }^{4}$ Health Economics Group, Maverex Limited, Manchester, UK

${ }^{5}$ Statistical Group, Maverex Limited, Manchester, UK

${ }^{6}$ Data Science \& Al, AstraZeneca, Gaithersburg, Maryland, USA

${ }^{7}$ Global Pricing and Market Access, AstraZeneca, Gaithersburg, Maryland, USA

${ }^{8}$ Global Medical Affairs, AstraZeneca, Cambridge, UK

${ }^{9}$ BioPharmaceuticals Medical, AstraZeneca, Gaithersburg, Maryland, USA

Acknowledgements Writing and editing assistance was provided by Sarah Thornburg of JK Associates Inc, a Fishawack Health Company. This support was funded by AstraZeneca.

Contributors NP, JL, LN, SL and EH designed the research. NP, JL, LN, SL and NE conducted the research. NP and NE performed the statistical analysis. NP drafted the manuscript. JL and SL supervised the writing. JY, NP, JL, LN, SL, NE, XW, BD, VB and EH contributed to the data interpretation and revised each draft for important intellectual content. All authors read and approved the final manuscript. EH had primary responsibility for the final content and is the guarantor. The corresponding author (BD) attests that all listed authors meet authorship criteria and that no others meeting the criteria have been omitted.

Funding The current analysis was supported by funding from AstraZeneca. The funder of the study had a role in its design, interpretation of the data and in the writing of the manuscript. The funder had no role in the conduct, collection or analysis of the data. All authors had full access to all of the data in the study and can take responsibility for the integrity of the data and the accuracy of the data analysis.

Competing interests All authors have completed the ICMJE uniform disclosure form at www.icmje.org/coi_disclosure.pdf and declare that NP, JL, LN, SL and NE have received personal fees from AstraZeneca during the conduct of the study and outside the submitted work. VB, XW, BD and EH are employees of AstraZeneca.

Patient consent for publication Not required.

Ethics approval Ethics approval was not required because the study involved only data from previous studies and did not involve obtaining new data from participants. No human subject participants were directly involved in this metanalysis.

Provenance and peer review Not commissioned; externally peer reviewed.

Data availability statement Data underlying the findings described in this manuscript may be obtained in accordance with AstraZeneca's data sharing policy described at https://astrazenecagrouptrials.pharmacm.com/ST/Submission/ Disclosure.

Supplemental material This content has been supplied by the author(s). It has not been vetted by BMJ Publishing Group Limited (BMJ) and may not have been peerreviewed. Any opinions or recommendations discussed are solely those of the author(s) and are not endorsed by BMJ. BMJ disclaims all liability and responsibility arising from any reliance placed on the content. Where the content includes any translated material, BMJ does not warrant the accuracy and reliability of the translations (including but not limited to local regulations, clinical guidelines, terminology, drug names and drug dosages), and is not responsible for any error and/or omissions arising from translation and adaptation or otherwise.

Open access This is an open access article distributed in accordance with the Creative Commons Attribution Non Commercial (CC BY-NC 4.0) license, which permits others to distribute, remix, adapt, build upon this work non-commercially, and license their derivative works on different terms, provided the original work is properly cited, appropriate credit is given, any changes made indicated, and the use is non-commercial. See: http://creativecommons.org/licenses/by-nc/4.0/

\section{REFERENCES}

1 Lopez R, Davidson JE, Beeby MD, et al. Lupus disease activity and the risk of subsequent organ damage and mortality in a large lupus cohort. Rheumatology 2012;51:491-8.

2 Cervera R, Khamashta MA, Font J, et al. Morbidity and mortality in systemic lupus erythematosus during a 10-year period. Medicine 2003;82:299-308.

3 Gladman DD. Prognosis and treatment of systemic lupus erythematosus. Curr Opin Rheumatol 1996;8:430-7.
4 Gladman D, Ginzler E, Goldsmith C, et al. The development and initial validation of the Systemic Lupus International Collaborating Clinics/ American College of Rheumatology damage index for systemic lupus erythematosus. Arthritis Rheum 1996;39:363-9.

5 Bernatsky S, Clarke A, Gladman DD, et al. Mortality related to cerebrovascular disease in systemic lupus erythematosus. Lupus 2006;15:835-9.

6 Bernatsky S, Boivin JF, Joseph L, et al. Mortality in systemic lupus erythematosus. Arthritis Rheum 2006;54:2550-7.

7 Björnådal L, Yin L, Granath F, et al. Cardiovascular disease a hazard despite improved prognosis in patients with systemic lupus erythematosus: results from a Swedish population based study 1964-95. J Rheumatol 2004;31:713-9.

8 Urowitz MB, Gladman DD, Tom BDM, et al. Changing patterns in mortality and disease outcomes for patients with systemic lupus erythematosus. J Rheumatol 2008;35:2152.

9 Lee YH, Choi SJ, Ji JD, et al. Overall and cause-specific mortality in systemic lupus erythematosus: an updated meta-analysis. Lupus 2016;25:727-34.

10 Schoenfeld SR, Kasturi S, Costenbader KH. The epidemiology of atherosclerotic cardiovascular disease among patients with SLE: a systematic review. Semin Arthritis Rheum 2013;43:77-95.

11 Yurkovich M, Vostretsova K, Chen W, et al. Overall and cause-specific mortality in patients with systemic lupus erythematosus: a meta-analysis of observational studies. Arthritis Care Res 2014;66:608-16.

12 Bhatnagar P, Wickramasinghe K, Wilkins E, et al. Trends in the epidemiology of cardiovascular disease in the UK. Heart 2016;102:1945-52.

13 Holmqvist M, Simard JF, Asplund K, et al. Stroke in systemic lupus erythematosus: a meta-analysis of population-based cohort studies. RMD Open 2015;1:e000168.

14 Pooley N, Langham J, Langham S, et al. The risk of major cardiovascular events in adult patients with systemic lupus erythematosus (SLE): systematic review and meta-analysis protocol. PROSPERO CRD42018098690 [updated 2018]. Available https:// www.crd.york.ac.uk/prospero/display record.php?ID= CRD42018098690 (accessed 18 Oct 2019).

15 Higgins J, Green S. Cochrane handbook for systematic reviews of interventions. Version 5.1.0 [updated March 2011]. London: The Cochrane Collaboration, 2011.

16 Stroup DF, Berlin JA, Morton SC, et al. Meta-analysis of observational studies in epidemiology: a proposal for reporting. Meta-analysis of Observational Studies in Epidemiology (MOOSE) group. JAMA 2000;283:2008-12.

17 Tan EM, Cohen AS, Fries JF, et al. The 1982 revised criteria for the classification of systemic lupus erythematosus. Arthritis Rheum 1982;25:1271-7.

18 Hochberg MC. Updating the American College of Rheumatology revised criteria for the classification of systemic lupus erythematosus. Arthritis Rheum 1997:40:1725.

19 Wells G, Shea B, O'Connell D, et al. The Newcastle-Ottawa Scale (NOS) for assessing the quality of nonrandomised studies in meta-analyses. 2014. Available from http://www.ohri.ca/programs/clinical_epidemiol ogy/oxford.asp (accessed 21 Oct 2019)

20 Avina-Zubieta JA, Thomas J, Sadatsafavi M, et al. Risk of incident cardiovascular events in patients with rheumatoid arthritis: a meta-analysis of observational studies. Ann Rheum Dis 2012;71:1524-9.

21 Avina-Zubieta JA, Choi HK, Sadatsafavi M, et al. Risk of cardiovascula mortality in patients with rheumatoid arthritis: a meta-analysis of observational studies. Arthritis Rheum 2008;59:1690-7.

22 Bhutta AT, Cleves MA, Casey PH, et al. Cognitive and behavioral outcomes of school-aged children who were born preterm: a meta-analysis. JAMA 2002;288:728-37.

23 Takkouche B, Etminan M, Montes-Martínez A. Personal use of hair dyes and risk of cancer. JAMA 2005;293:2516.

24 Greenland S. Quantitative methods in the review of epidemiological literature. Epidemiol Rev 2017;9:1-30.

25 DerSimonian R, Laird N. Meta-analysis in clinical trials. Control Clin Trials 1986;7:177-88.

26 Stuck AE, Rubenstein LZ, Wieland D, et al. Bias in meta-analysis detected by a simple, graphical. BMJ 1998;316:469-69.

27 Viechtbauer W. Conducting meta-analyses in R with the metafor package. J Stat Softw 2010;36:1-48.

28 Arkema VE, Svenungsson E, Von Euler M, et al. Stroke in systemic lupus erythematosus: a Swedish population-based cohort study. Ann Rheum Dis 2017;76:1544-9.

29 Avina-Zubieta JA, To F, Vostretsova K, et al. Risk of myocardial infarction and stroke in newly diagnosed systemic lupus erythematosus: a general population-based study. Arthritis Care Res 2017;69:849-56. 
30 Bengtsson C, Ohman ML, Nived O, et al. Cardiovascular event in systemic lupus erythematosus in northern Sweden: incidence and predictors in a 7-year follow-up study. Lupus 2012;21:452-9.

31 Chang Y-SS, Liu C-JJ, Chen W-SS, et al. Increased risk of subarachnoid hemorrhage in patients with systemic lupus erythematosus: a nationwide population-based study. Arthritis Care Res 2013;65:601-6.

32 Chiu CC, Huang CC, Chan WL, et al. Increased risk of ischemic stroke in patients with systemic lupus erythematosus: a nationwide population-based study. Intern Med 2012;51:17-21.

33 Dregan A, Chowienczyk P, Molokhia M. Cardiovascular and type 2 diabetes morbidity and all-cause mortality among diverse chronic inflammatory disorders. Heart 2017;103:1867-73.

34 Faurschou M, Mellemkjaer L, Starklint H, et al. High risk of ischemic heart disease in patients with lupus nephritis. J Rheumatol 2011;38:2400-5.

35 Hak AE, Karlson EW, Feskanich D, et al. Systemic lupus erythematosus and the risk of cardiovascular disease: results from the nurses' health study. Arthritis Rheum 2009;61:1396-402.

36 Hermansen ML, Lindhardsen J, Torp-Pedersen C, et al. The risk of cardiovascular morbidity and cardiovascular mortality in systemic lupus erythematosus and lupus nephritis: a Danish nationwide population-based cohort study. Rheumatology 2017;56:709-15

$37 \mathrm{Kim} \mathrm{CH}$, Al-Kindi SG, Jandali B, et al. Incidence and risk of heart failure in systemic lupus erythematosus. Heart 2017;103:227-33.

38 Krishnan E. Stroke subtypes among young patients with systemic lupus erythematosus. Am J Med 2005;118:1415.

39 Lin CY, Shih CC, Yeh CC, et al. Increased risk of acute myocardial infarction and mortality in patients with systemic lupus erythematosus: two nationwide retrospective cohort studies. Int J Cardiol 2014;176:847-51.

40 Liou TH, Huang SW, Lin JW, et al. Risk of stroke in patients with rheumatism: a nationwide longitudinal population-based study. Sci Rep 2014;4:5110.

41 Manzi S, Meilahn EN, Rairie JE, et al. Age-specific incidence rates of myocardial infarction and angina in women with systemic lupus erythematosus: comparison with the framingham study. Am J Epidemiol 1997;145:408-15.

42 Mok CC, Ho LY, To CH. Annual incidence and standardized incidence ratio of cerebrovascular accidents in patients with systemic lupus erythematosus. Scand J Rheumatol 2009;38:362-8.

43 Ramagopalan VS, Pakpoor J, Seminog O, et al. Risk of subarachnoid haemorrhage in people admitted to hospital with selected immune-mediated diseases: record-linkage studies. BMC Neurol 2013;13:176.

44 Rees F, Doherty M, Grainge M, et al. Burden of comorbidity in systemic lupus erythematosus in the UK, 1999-2012. Arthritis Care Res 2016;68:819-27.

45 Wang IK, Muo CH, Chang YC, et al. Risks, subtypes, and hospitalization costs of stroke among patients with systemic lupus erythematosus: a retrospective cohort study in Taiwan. J Rheumatol 2012;39:1611-18

46 Zoller B, Li X, Sundquist J, et al. Risk of subsequent ischemic and hemorrhagic stroke in patients hospitalized for immune-mediated diseases: a nationwide follow-up study from Sweden. BMC Neurol 2012;12:41.

47 Ward MM. Premature morbidity from cardiovascular and cerebrovascular diseases in women with systemic lupus erythematosus. Arthritis Rheum 1999;42:338-46.

48 Barnado A, Carroll RJ, Casey C, et al. Phenome-wide association study identifies marked increased in burden of comorbidities in African Americans with systemic lupus erythematosus. Arthritis Res Ther 2018;20:69.

49 Cook MJ, Bellou E, Bowes J, et al. The prevalence of co-morbidities and their impact on physical activity in people with inflammatory rheumatic diseases compared with the general population: results from the UK Biobank. Rheumatology (Oxford) 2018;57:2172-82.

$50 \mathrm{Lim}$ SY, Bae EH, Han KD, et al. Systemic lupus erythematosus is a risk factor for cardiovascular disease: a nationwide, population-based study in Korea. Lupus 2018;27:2050-6.

51 Fanouriakis A, Kostopoulou M, Alunno A, et al. 2019 update of the EULAR recommendations for the management of systemic lupus erythematosus. Ann Rheum Dis 2019;78:736-45.

52 Olesińska M, Saletra A. Quality of life in systemic lupus erythematosus and its measurement. Reumatologia 2018;56:45-54.

53 Fors Nieves CE, Izmirly PM. Mortality in systemic lupus erythematosus: an updated review. Curr Rheumatol Rep 2016;18:21.

54 Mok CC. Accelerated atherosclerosis, arterial thromboembolism, and preventive strategies in systemic lupus erythematosus. Scand $J$ Rheumatol 2006;35:85-95.

55 Sari RM, Kasjmir YI, Antono D, et al. Atherosclerosis prevalence and the correlation between atherosclerosis risk factors and carotid intima-media thickness in below 40-year-old women with systemic lupus erythematosus. Indonesian J Rheumatol 2010;3:24-30.

56 Gartshteyn Y, Braverman G, Mahtani S, et al. Prevalence of coronary artery calcification in young patients with SLE of predominantly Hispanic and African-American descent. Lupus Sci Med 2019;6: e000330.

57 Seligman VA, Lum RF, Olson JL, et al. Demographic differences in the development of lupus nephritis: a retrospective analysis. Am J Med 2002;112:726-9.

58 Gustafsson JT, Herlitz Lindberg M, Gunnarsson I, et al. Excess atherosclerosis in systemic lupus erythematosus,-A matter of renal involvement: case control study of 281 SLE patients and 281 individually matched population controls. PLoS ONE 2017;12:e0174572.

59 González LA, Alarcón GS, Gonzalez LA, et al. The evolving concept of SLE comorbidities. Expert Rev Clin Immunol 2017;13:753-68.

60 Ajeganova S, Hafström I, Frostegåd J. Risk of CV events and mortality in SLE is associated with accumulated disease-damage, anti-phospholipid syndrome and higher carotid intima-media thickness. Lupus Sci Med 2020;7:A107-8. 